\title{
Vivre entre deux cultures frontalières : comment un habitat multiethnique oriente l'auto- identification
}

Life on the Border Area or How the Multicultural Environment Dictates the Rules of Self-identification

\section{Roza Laptander}

Traducteur : Eva Toulouze

\section{OpenEdition}

Journals

Édition électronique

URL : https://journals.openedition.org/efo/2552

DOI : $10.4000 /$ efo. 2552

ISSN : 2275-1947

Éditeur

INALCO

Édition imprimée

Date de publication : 1 décembre 2013

ISBN : 978-2-343-04446-0

ISSN : 0071-2051

Référence électronique

Roza Laptander, «Vivre entre deux cultures frontalières : comment un habitat multiethnique oriente l'auto-identification », Études finno-ougriennes [En ligne], 45 | 2013, mis en ligne le 12 février 2015, consulté le 08 juillet 2021. URL : http://journals.openedition.org/efo/2552 ; DOI : https://doi.org/ 10.4000/efo.2552

Ce document a été généré automatiquement le 8 juillet 2021.

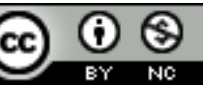

Études finno-ougriennes est mis à disposition selon les termes de la Licence Creative Commons Attribution - Pas d'Utilisation Commerciale 4.0 International. 


\title{
Vivre entre deux cultures
}

\section{frontalières : comment un habitat multiethnique oriente l'auto- identification $^{1}$}

\author{
Life on the Border Area or How the Multicultural Environment Dictates the Rules \\ of Self-identification
}

Roza Laptander

Traduction : Eva Toulouze

\section{Introduction}

1 Je voudrais évoquer, en commençant cet article, le titre d'un article d'Ariadne Kuznecova, linguiste samoyédologue moscovite qui, dans son article «La variance comme facteur de dislocation du système linguistique dans le processus de mutation linguistique (sur des matériaux selkoupes) », parle de cultures hybrides chez les Khantys, les Mansis et les Nénets (Kuznecova 2007, p. 140). Ce titre me semble convenir à merveille pour décrire la situation linguistique des Nenets de l'Oural qui ont des racines khantyes. Il pourrait tout autant se rapporter aux Khantys qui ont intégré la culture nenets, et ne peuvent donc que difficilement se définir par leur culture et ethnie d'origine.

2 Les mélanges de langues et de cultures sont à l'ordre du jour, bien que dans la plupart des cas il en soit question à propos de la langue et de la culture d'un peuple puissant qui en écrase un plus faible. J'ai écrit moi-même quelques travaux à ce sujet, mais avec le temps, je me dis, à ma grande honte, que la langue dans laquelle je les ai écrits était trop sèche ; je n'y présentais qu'une constatation nue de certains faits, sans forcément les illustrer par un tableau de la réalité. Ici, je voudrais introduire mes pensées, impressions et souvenirs, afin d'élargir le cadre de cet article, et ainsi rendre compte 
d'une image intériorisée de la réalité. Trop souvent, ce qu'on voit ou entend à l'intérieur de sa propre culture à son propos ${ }^{2}$ est accepté comme une évidence ; on ne le met pas en question, on n'en recherche pas les causes. Ce n'est qu'avec les années, en analysant les événements du passé, qu'on en comprend mes raisons.

\section{Vivre dans un arrondissement multiethnique}

3 L'arrondissement autonome des Nenets du Jamal est le $89^{\mathrm{e}}$ sujet de la Fédération de Russie. Il est situé au nord de la Sibérie occidentale. Il est multiethnique. La population immigrée est ici très nombreuse : elle a commencé à s'installer dans le nord dans les années 1980 et 2000. La plupart des migrants sont venus de Russie centrale, d'Ukraine ainsi que de diverses républiques de l'ancienne Union soviétique.

4 Ces migrations se poursuivent, la population augmente. C'est un processus particulièrement actif ces temps-ci en raison de l'exploitation du gaz dans la péninsule du Jamal ; de plus, toutes sortes d'entreprises se créent dans les différentes branches du complexe pétrolier. Cet arrondissement est en effet considéré comme l'une des régions les plus fabuleusement riches de la Russie, avec des salaires élevés et des primes complémentaires considérables, en compensation pour les conditions extrêmes du Grand Nord pour la santé des travailleurs. Ceux-ci viennent travailler ici pour améliorer leur position financière. Beaucoup de migrants prévoient de rester un ou deux, pas plus ; souvent, de fait, ils restent bien plus longtemps. Ils finissent par obtenir des appartements, fondent une famille, jettent des racines; il devient de plus en plus difficile pour eux de rentrer dans leurs contrées d'origine.

La population autochtone se compose de Nenets, de Khantys et de Selkoupes. Tous ensemble, ils représentent $8,2 \%$ de la population de l'arrondissement. On peut suivre dans le tableau ci-dessous l'évolution de la structure ethnique de la région:

\begin{tabular}{|l|l|l|l|l|l|l|l|l|}
\hline \multirow{2}{*}{$\begin{array}{l}\text { Groupe } \\
\text { ethnique }\end{array}$} & \multicolumn{2}{l}{$\begin{array}{l}\text { Recensement de } \\
1979\end{array}$} & \multicolumn{2}{l}{$\begin{array}{l}\text { Recensement de } \\
1989\end{array}$} & \multicolumn{2}{l}{$\begin{array}{l}\text { Recensement de } \\
2002\end{array}$} & $\begin{array}{l}\text { Recensement de } \\
2010\end{array}$ & \\
\cline { 2 - 9 } & nombre & $\%$ & nombre & $\%$ & nombre & $\%$ & nombre & $\%$ \\
\hline Nenets & 17404 & $11,0 \%$ & 20917 & $4,2 \%$ & 26435 & $5,2 \%$ & 29772 & $5,9 \%$ \\
\hline Khantys & 6466 & $4,1 \%$ & 7247 & $1,5 \%$ & 8760 & $1,7 \%$ & 9489 & $1,9 \%$ \\
\hline Komis & 5642 & $3,6 \%$ & 6000 & $1,2 \%$ & 6177 & $1,2 \%$ & 5141 & $1,0 \%$ \\
\hline Selkoupes & 1611 & $1,0 \%$ & 1530 & $0,3 \%$ & 1797 & $0,4 \%$ & 1988 & $0.4 \%$ \\
\hline Russes & 93750 & $59,0 \%$ & 292808 & $59,2 \%$ & 298359 & $588 \%$ & 312019 & $61,7 \%$ \\
\hline Ukrainiens & 15721 & $9,9 \%$ & 85022 & $17,2 \%$ & 66080 & $13,0 \%$ & 48985 & $9,7 \%$ \\
\hline Tatars & 8556 & $5,4 \%$ & 26431 & $5,3 \%$ & 27734 & $5,5 \%$ & 28509 & $5,6 \%$ \\
\hline Autres & 9694 & $6,1 \%$ & 54889 & $11,1 \%$ & 71664 & $14,1 \%$ & 74625 & $14,3 \%$ \\
\hline
\end{tabular}

6 Ces chiffres diffèrent sensiblement des données démographiques collectées en 1933-1935. Dans le rapport sur l'expédition consacrée à l'étude de l'aménagement du rajon Priural'skij, on trouve les chiffres et commentaires suivants : 
Dans le rajon, les plus nombreux sont les Nenets, qui représentent $33 \%$ de la population totale; ils sont suivis par les Khantys, avec $32 \%$, en troisième position viennent les Zyriènes, avec $28 \%$; les Russes sont au bas de la liste avec $7 \%$. Il faut noter aussi que la grande masse de la population russe, près de $90 \%$, et une partie considérable des Zyriènes, $50 \%$, sont concentrés dans le chef-lieu de l'arrondissement, le bourg de Sale-Hard, qui se situe dans ce même rajon; si on le laisse de côté, le poids respectif des populations change considérablement. Quant au poids des différentes nationalités dans les soviets nationaux, nous constatons que le poids des Nenets est particulièrement important dans le soviet de Ščuč'erečje, où ils représentent $63 \%$ de la population, et il est le moins grand dans le soviet de Sob, où ils ne sont que $78 \%$. La population khantye prédomine dans les soviets de Toupogol' et de Sob. Dans le premier, ils sont 63,55 \%, dans le deuxième 64,48 \%. Dans le soviet nomade de Ščuč'erečje ils ne sont que $24,41 \%$. Les Zyriènes et les Russes sont en majorité à Salehard, comme nous l'avons déjà dit (1935, p. 51).

7 Aujourd'hui, on peut dire que dans la vie d'un bourg pluriethnique les frontières entre les nationalités sont estompées : les Nenets et les Khantys ne se présentent plus comme des groupes ethniques distincts. Pour beaucoup d'habitants des bourgs, surtout pour les enfants des familles russophones, il est compliqué de distinguer les nationalités mentionnées ci-dessous l'une de l'autre. C'est pourquoi il est plus simple pour eux de faire appel à une représentation globale des peuples du Nord, tels qu'ils se trouvent présentés dans les illustrations des livres pour enfants, des manuels, des cartes de nouvel an (cf. ci-dessous).

Firsanova I. éd. Arts graphiques, URSS, 1988

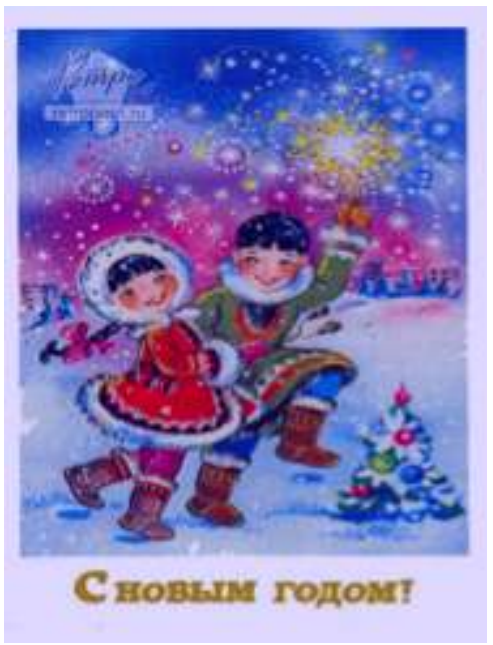

Bien sûr, cela ne voulait pas dire que le mot čukča, utilisé dans toute la Russie ${ }^{3}$ (et l'Union soviétique), fût utilisé fréquemment, mais on racontait bien des blagues à leur sujet.

Il faut noter que la population immigrée russophone des grandes villes de l'arrondissement des Nenets du Jamal appelle les ressortissants des peuples du Nord "nationaux » (nacionaly). Ce terme est issu du nom des arrondissements nationaux ${ }^{4}$. Ici, on utilise aussi un autre terme, encore plus dépréciatif et méprisant, pour les autochtones : ХАНТ (hant) ou ХАНТЭЙКА (hantejka ${ }^{5}$ ). Ce terme, pour les enfants de la population russophone immigrée qui s'adressent aux enfants autochtones de leur âge, est chargé du sens de " primitif, sauvage, idiot », ce qui est plus fort que $l^{6}{ }^{6}$ " bête ». 
10 Tout d'abord, je dois préciser que j'ai passé toute ma petite enfance dans la toundra de Bajdarata, dans le rajon Priural'skij de l'arrondissement des Nenets du Jamal, qui a toujours été une zone frontière entre les territoires des Khantys et des Nenets, surtout dans les régions du bas cours de l'ob et du Poluj. Ces terres, surtout à proximité de la ville de Salehard, étaient un lieu de rencontre, de commerce, d'échange, et, dans un passé reculé, de confrontation entre clans ennemis de Khantys et de Nenets. Le folklore des deux peuples garde le souvenir d'affrontements armés les uns contre les autres, où les perdants étaient souvent faits prisonniers. Ces affrontements représentaient le stade ultime de conflits territoriaux, dans la mesure où les larges berges de l'ob étaient un excellent terrain de pêche et de chasse. Dans le folklore des Nenets, la plupart de ces événements sont situés sur les rives de "la rivière aux saules ", ce qui est la traduction $\mathrm{du}$ terme nenets pour désigner l'ob. Ces conflits finirent par être résolus. Les deux communautés firent la paix et vécurent à proximité l'une de l'autre, scellant leur accord par un traité de paix : le territoire fut partagé de telle sorte que la rive droite de l'Ob revenait aux Khantys, alors que la rive gauche septentrionale devenait territoire nenets.

11 Mon rapport individuel à ma culture nenets s'est formé de manière entièrement chaotique. Bien que je sois née et que j'aie passé une partie de mon enfance dans la toundra, j'ai été aussi au jardin d'enfants, puis j'ai été confiée à une classe de village où j'ai fait mes études avec des enfants de familles russes. J'ai fait face à ma position par un haut niveau de réussite, c'est pourquoi même en cas de conflit, ceux-ci se résolvaient avec l'aide des adultes. En même temps, il arrivait que tel ou tel groupe d'enfants issus de familles malheureuses m'insulte ou m'humilie. C'était, je pense, afin de relever leur propre appréciation de eux-mêmes et leur place dans le groupe. Il y a eu ainsi dans mon enfance un moment où j'ai dissimulé tout lien avec ma culture nenets. Si mes camarades me demandaient si je parlais nenets, je répondais que je ne connaissais aucune autre langue que le russe. Ce qui n'était pas vrai; mais cela facilitait les relations et me rapprochait de la culture du village.

12 L'été, une nouvelle vie commençait. Avec l'arrivée du premier hélicoptère partant pour la toundra, je rentrais chez mes parents. Les premiers jours, c'était toujours difficile de me remettre au rythme de la vie dans la toundra. Cela se passait pourtant sans à-coups, sans doute grâce à mes parents, qui répondaient avec philosophie à toute manifestation de caractère de leur enfant qui venait de ce qu'on appelait « la civilisation ». C'est ainsi que pour les enfants du bourg, j'étais « une des leurs »; mais je n'étais pas si différente des enfants de la toundra, j'étais aussi « une des leurs».

13 À la fin de la période soviétique, à la fin des années 1980 , mes parents, l'été et l'automne, pêchaient. Nous vivions alors dans un petit village de pêcheurs, qu'on appelait Embouchure du petit Ob (Ust'e Maloj Obi). Ici, il n'y avait d'autres bâtiments que quatre cabanes en rondins et des tentes nenets. Les habitants étaient d'une part des Khantys qui y vivaient toute l'année, d'autre part des Nenets sans rennes, que mes parents appelaient, non sans arrogance, ЁДЭЙ ТЕР", c'est-à-dire « Nenets sédentaires ", lesquels, souvent, buvaient. Notre famille était différente: mes parents passaient l'hiver dans la toundra et ils avaient des rennes. Ils étaient rattachés au sovkhoze de Bajdarata et au bourg de Belojarsk, avaient un salaire qui leur permettait d'acheter de la nourriture pour l'hiver ; l'automne, ils faisaient des réserves de poisson salé, qui leur permettaient sous-traiter ceux qui s'occupaient de leurs rennes pendant l'été. 

qui remonte à la période soviétique : pour élever la productivité du travail, des Nenets "privés » furent associés au travail des entreprises de pêche de la région. Pour eux, cette activité était nouvelle, mais à l'époque ils avaient un besoin cuisant de revenus et donc, comme bien d'autres familles possédant peu de rennes, ils durent effectuer tous les ans la longue traversée depuis la toundra de Ščučerečje jusqu'au bas cours de l'Ob. Peu à peu, cette activité devint source de revenus stables, c'est pourquoi beaucoup de Nenets se mirent à passer l'été sur les lieux de pêche de l'ob. Ils y vivaient côte à côte avec des pêcheurs khantys; en hiver et au printemps, alors qu'ils étaient avec les rennes, ils avaient davantage de rencontres avec les Komis-Zyriènes.

intéressantes et suscitaient beaucoup d'émotions et de questions. La culture des peuples voisins, leur langue, qui était si différente du nenets à l'oreille, leurs vêtements et leurs règles de comportement suscitaient une approche prudente, mais dépourvue de toute agressivité ; c'était un intérêt salutaire.

conversations me firent comprendre que ces mêmes Khantys et Komis-Zyriènes étaient les uns et les autres divisés en deux groupes : il y avait des Khantys, des demikhantys, et des ЕДЭЙ ТЕР", des Khantys sédentaires appauvris, qui avaient partiellement adopté la culture du village, mais qui n'étaient pas acceptés d'elle alors même qu'ils avaient rejeté la vie dans la toundra; il y avait des Komis-Zyriènes tout court et des НЕНЭЙ НЕНЭцЯ НЫСмА, c'est à dire des Zyriènes aux racines nenets. Souvent, les noms de famille khanty avaient des équivalents nenets, et vice-versa. Ce mélange des noms de famille nenets et khantys, leur dualité locale est fort complexe et interconnectée. Certes, le stéréotype veut que lorsqu'on a un nom de famille nenets, on est nenets, quand on a un nom de famille khanty, on est khanty; mais dans ce cas, ce n'est pas aussi simple. Souvent on découvre que des gens au nom de famille khanty se considèrent comme nenets.

17

Comment ceci a-t-il pu se produire? Les Khantys et les Nenets,et plus tard les KomiZyriènes ont vécu tellement longtemps côte à côte, que ceci s'est reflété dans leur culture et dans leur langue. Aujourd'hui, la question est fort complexe : il est difficile de savoir qui est exclusivement khanty ou exclusivement Nenets du Bas Ob, tant les emprunts mutuels sont nombreux. Pour ce qui est du lexique, il fait noter l'abondance des emprunts au russe, mais ici ce qui nous intéresse, ce sont les emprunts à l'intérieur même de ces groupes ethniques. Il est vrai que même $\mathrm{N}$. M. Tereščenko a noté que certains emprunts au russe dans le dialecte du Jamal ont une forme phonique proche des dialectes khantys du nord: il est possible qu'à l'origine ces emprunts ont été le résultat de contacts entre voisins (Tereščenko 1959). Ces phénomènes ne sont pas fortuits, dans la mesure où les Nenets vivant à l'est de l'Oural et parlant les dialectes du Jamal et Priuralskij, à la différence des autres groupes de Nenets, ont tioujours eu beaucoup de contacts avec les Khantys. La littérature spécialisée le mentionne souvent, cf. les travaux de Tereščenko, Barmič, Ljublinskaja. Ces contacts remontent à une période ancienne, dans la mesure où les sources russes des $\mathrm{XVI}^{\mathrm{e}}-\mathrm{XVII}{ }^{\mathrm{e}}$ siècles mentionnent ces populations vivant au nord et à l'est de l'Oural. Ainsi, dans «La Chronique des temps passés ", les Samoyèdes sont présentés comme étant les voisins septentrionaux des Ougriens de l'Ob. Il s'agissait visiblement de Nenets. Il est possible qu'à l'époque les Nenets aient occupé des territoires voisins de ceux habités par les 
Ougriens au Nord de l'Oural. Ainsi, on peut supposer que les premiers contacts entre Nenets et Khantys ont eu lieu au tournant du $\mathrm{I}^{\mathrm{e}}$ et du $\mathrm{II}^{\mathrm{e}}$ millénaire.

Dans son ouvrage "Matériaux et recherches sur le nenets", Natalija Mitrofanovna Tereščenko fait référence à l'existence en nenets de mots communs au nenets et au khanty et concernant l'élevage du renne et certains aspects de la culture matérielle. Dans la mesure où l'élevage du renne est une activité à proprement parler nenets qu'ils l'ont portée à une large échelle, de manière générale on conclut que ces terlmes ont été empruntés par les Khantys au nenets (Tereščenko 1956, p 238). Natalija Tereščenko explique dans ses travaux que l'existence de lexèmes communs au nenets et au khanty n'est pas le fruit du hasard, puisque les Nenets du Jamal et de l'Oural ont été et sont toujours en contact étroit avec les Khantys. Ces contacts ont eu des effets essentiels sur l'économie, la vie quotidienne ainsi que la langue (Tereščenko 1959, p. 97). Elle en déduit que les Khantys, qui dans le passé se sont installés sur des territoires habités par des Nenets, ont été assimilés par ces derniers.

À ce point, il convient de citer les mots de V. F. Zuev : «La terre des Samoyèdes est la dernière vers le nord. Elle est sauvage dans son appellation et dans son état, elle est habitée par des peuples différents et sauvages. Parmi eux, on compte deux lignées dans l'uezd de Berezovo, c'est-à-dire les Ostiaks et les Samoyèdes, mais les premiers vivent plus loin des berges de l'Océan arctique, et, installés loin sur les rives de l'ob, ils rejoignent les peuples de la Russie » (Zuev 1947, p. 21).

Plus loin, il écrit : « Mais les samoyèdes qui se sont installés en partie à proximité de l'embouchure de l'ob pour s'adonner à la pêche, sont comme des intermédiaires entre les deux peuples ${ }^{7}$, ils ont une langue particulière et des coutumes particulières, qu'il n'est pas nécessaire de décrire ici dans le détail et dans leurs spécificités; mais on peut comprendre même sans précisions que mêlant ainsi les deux peuples, les deux ont assimilé les pratiques et les coutumes, proches de celles des Samoyèdes et proches de celles des Ostiaks; avec les Samoyèdes, ils se comportent à la Samoyède, ave les Ostiaks comme les Ostiaks... Et ils prennent des femmes chez les uns et chez les autres, ce qui pose des problèmes non des moindres (idem, p. 52). Tereščenko estime que Zuev parle ici des clans d'origine khanty, qui ont subi une grande influence de la part des Nenets.

21 Les observations de cet ethnographe sont uniques, car les particularités des contacts entre Nenets et Khantys existent jusqu'à nos jours dans le Bas Ob, sur un petit territoire entre Salehard et jar Salé, avec des bourgs importants comme Aksarka, Zelenyj Jar, Tiovopogol, Belojarsk, Salemal, Ščuč'e et les petits villages de pêcheurs à proximité, proches des toundras du Poluj, de Bajdarata, de Laborobo et de Jamal. C'est ici que l'on note le plus grand nombre de mariages mixtes, et par conséquent, c'est ici que le bilinguisme est le plus répandu dans les familles. On peut présenter un certain nombre d'exemples de familles nenets ayant adopté la culture et la langue khanty et de familles khantys parlant nenets, nomadisant dans la toundra tout en suivant leurs propres traditions.

Parmi les familles de la péninsule du Jamal, il faut noter les noms de Nerkagi, de Pando, de Porunguj, de Salinder et de Tibiči (idem, p.98). On peut les analyser sur la base du nenets. Ceux qui portent ces noms vivent depuis longtemps sur le territoire du rajon Priuralskij. Ils considèrent le nenets comme leur langue maternelle, vient comme des Nenets, nomadisent avec les rennes, beaucoup d'entre eux pêchent, comme bien des familles nenets. Et pourtant, les Nenets les considèrent comme khantys, car ils sont d'origine khanty. Dans la mesure où dans beaucoup de famille on trouve des coutumes 
différentes de celles des Nenets, on les rapproche plutôt de la culture khantye. Ils connaissent des interdits de consommation de l'esturgeon par les femmes, alors que chez les Nenets on rencontre plutôt l'interdit de consommation du brochet. De même qu'il est interdit pour les femmes de couper et de nettoyer le brochet, elles ne peuvent pas se livrer à ces activités sur la lotte. Les personnes portant les noms de famille mentionnés ont des relations familiales avec les Khantys du Bas Ob, et certaines d'entre elles parlent mieux khanty que nenets; beaucoup portent deux noms de famille, l'un khanty et l'autre nenets. De plus, si les Khantys qui habitent depuis longtemps en milieu nenets ont assimilé le nenets et certaines coutumes, ils n'en gardent pas moins certaines de leurs caractéristiques ethniques.

Curieusement, leurs familles les considèrent comme Khantys, alors que pour les Khantys ils sont Nenets. Leur folklore familial a préservé des légendes sur leur histoire nenets, et pourtant ils s'identifient comme khantys. Mais tout ceci s'est passé dans une période récente, pas plus qu'il y a trois générations.

Souvent, les noms de famille khantys avaient des équivalents nenets, et vice-versa. $\mathrm{Ce}$ mélange des noms de famille nenets et khantys, leur dualité locale est fort complexe et interconnectée. Certes, le stéréotype veut que lorsqu'on a un nom de famille nenets, on est nenets, quand on a un nom de famille khanty, on est khanty ; mais dans ce cas, ce n'est pas aussi simple. Souvent on découvre que des gens au nom de famille khanty se considèrent comme nenets. L'explication était la suivante : les personnes vivant dans le rajon Priural'skij et à ses frontières avec le rajon du Jamal le long du bas Ob, présentent souvent dans les conversations et dans les narrations des légendes sur leur origine, qu'elle soit khanty ou nenets. D'ailleurs, les narrateurs eux-mêmes s'identifient en tant que Khantys ou Nenets. Cette mutation culturelle, ce passage d'une identité à l'autre, a eu lieu à une époque relativement récente, peut-être il y a trois ou quatre générations.

Un exemple est ainsi le clan portant le nom de famille de Venengo. L'exemple opposé est celui des Salinder, qui sont d'origine khantye, mais dont les membres se considèrent traditionnellement nenets. Au sud du rajon Priural'skij on trouve abondamment les noms de famille nenets Negači, Nerkagi, Poronguj, Salinder, Tibiči. La population locale les considère comme des Khantys. Comme l'avait déjà fait remarquer N. M. Tereščenko, ces familles ont des liens de parenté avec les Khantys du bas Ob ; certains d'entre eux maitrisent mieux le khanty que le nenets, et de plus beaucoup portent deux noms de famille, l'un nenets et l'autre khanty (Tereščenko 1956, p. 98). En plus du khanty, la connaissance du nenets est largement répandue dans ces familles. Dans la littérature spécialisée, ce groupe est souvent appelé « de l'Ob-Poluj ", dans la mesure où « vivant en étroite proximité avec les Nenets de l'Oural et du Jamal, cela fait longtemps que les deux groupes se sont mélangés, débouchant depuis longtemps sur une culture unique, dont ils sont les seuls porteurs » (Sjazi 2000, p. 98).

Dans la toundra de Panaevo vivent des Klimov (une variante des Nerkagi, les gens des saules, vivant dans des endroits où poussent les saules, c'est-à-dire le long de rivières ou de ruisseaux), des Salinder (habitants des berges des fleuves, des promontoires), des Kondygin, dont l'équivalent nenets est Lohorta (ruisseau, petit ruisseau) - ce sont des Khantys, alors que tous les autres, ce sont des Nenets enregistrés sous le nom de Negači. 
Dans le bourg de Panaevsk j'ai rencontré une famille khanty. Quand nous avons fait connaissance, je leur ai demandé leur nom de famille et le père de famille a répondu qu'ils s'appelaient Lohorta. Je n'avais jamais entendu ce nom, je lui ai reposé la question et il m'a donné la même réponse. Alors sa femme m'a expliqué qu'ils étaient des Kondygin, des Khantys, mais qu'à la maison ils ne parlent que nenets. Le désir même du chef de famille de prononcer la version nenets de son nom reflétait sans doute son désir de se rapprocher de la culture nenets, et de ne pas être isolé de la majorité de la population nenets, puisque j'en étais et que je parlais nenets avec lui.

Le nom nenets Padranhasovy (пэДАРА' ХАСАВА" en nenets «homme de la forêt ») est interprété par les Nenets come étant khanty.

Beaucoup de Khantys sont passés au nenets à un âge avancé. Ceci peut s'expliquer par diverses raisons, mais la première est le mariage. C'est le cas d'ol'ga Kujvina, qui vit au bourg de Panaevsk, et qui a appris le nenets quand elle s'est mariée avec un éleveur de rennes khanty du rajon voisin de Jamal. Néanmoins, tous ses enfants parlent khanty en famille.

En famille on parle khanty et nenets. La femme de mon fils, une Horolja, est nenets. Elle est de la toundra de Panaevo, on a fait la noce suivent les anciennes traditions nenets. Alors qu'on préparait la noce, sa mère a dit: "Les enfants ne savent pas comment étaient les noces traditionnelles, montrons-leur comment faire !» Comme elle est nenets, elle a tout fait suivant les traditions nenets, et nous, après, on a fait la noce à la khanty. Ma deuxième belle-fille est nenets, elle aussi.

Dans leur famille, on parle en permanence les deux langues. La mère d'ol'ga était nenets, une Vylka du rajon Priural'skij, et après son mariage, elle a parlé à ses enfants en nenets. La famille de la grand-mère d'ol'ga nomadisait dans les montagnes, et après la mort de son premier mari, elle a épousé un Sjadaev. Sa famille continue à vivre dans le village de Ščuče, dépendant du bourg de Belojarsk.

3 Alors à quel groupe ethnique se rattachent les personnes qui sont nées et qui ont grandi dans des familles mixtes? Quand j'ai demandé à ol'ga avec quel groupe ethnique elle s'identifie, elle m'a dit qu'elle se sentait plutôt khanty, et je me suis dit que pour moi aussi, de ce fait, elle est khanty, bien que sa mère soit nenets. On voit bien que la domination de l'une des cultures sur un territoire donné détermine jusqu'à la langue dans la famille. Dans les endroits où est implantée majoritairement une population parlant nenets, c'est le nenets qui domine, et même les familles khantys se mettent à utiliser cette langue. Mais il peut arriver aussi que de nombreuses familles utilisent à l'intérieur de la famille le khanty, sans que la langue voisine n'interfère.

4 Comme ol'ga elle-même le dit, ces derniers temps, même en vivant dans la toundra, ils mélangent le russe et le khanty. Même les enfants en font souvent la remarqué à leur mère : au lieu d'utiliser des mots khantys, elle fait appel à l'équivalent russe. Je lui ai demandé pourquoi. ol'ga a répondu alors que parmi les jeunes, personne ne chante plus les chants personnels, tous chantent du pop russe. Ils regardent des films en vidéo, surtout dans le rajon Priural'skij: autour du bourg de Belojarsk on trouve des magnétoscopes dans toutes les tentes (Kyjbina O. L. 2006).

Analysons à l'aide d'un schéma comment se forment ces groupes mixtes.

Mère khanty + père nenets $=$ enfant nenets

Mère nenets + père khanty $=$ enfant khanty 
37 En même temps, nombreux sont les enfants de couples mixtes qui se rattachent chacun à une ethnie différente, ce qui la plupart du temps tient aux liens qu'ils ont avec leurs parents. Souvent, les filles se sentent plus proches de leur mère, les fils de leur père. Souvent, le changement de langue et d'identité se produit sous l'effet de plusieurs facteurs. Dans son ouvrage "Les langues des peuples du Nord au xx siècle", N. B. Vahtin formule des prévisions tout à fait pessimistes sur l'avenir des langues du Nord. Néanmoins, son analyse des principaux facteurs qui influent négativement sur la vitalité des langues est fort pertinente: le nombre de locuteurs, les groupes d'âge, le caractère des mariages (mixtes), l'éducation des enfants dans les internats, le lieu d'habitation, les contacts linguistiques, l'existence des modes de vie traditionnels, la conscience nationale.

39 Comme l'a fait remarquer N. B. Vahtin, plus les locuteurs sont nombreux, moins il est vraisemblable qu'une langue sorte de l'usage. Sur d'autres questions, il renvoie à un autre sociolinguiste, A. E. Kibrik, d'après lequel plus les contacts avec d'autres langues sont nombreux, moins la langue en question a de vitalité, et il complète son affirmation aussitôt en ajoutant que «cette dépendance est plus complexe: le bilinguisme ou trilinguisme d'une région dans la plupart des cas n'empêche pas la communauté examinée de parler sa langue à l'intérieur de son groupe » (Vahtin 2001, p. 224).

40 Mais d'autre part, une enquête orale menée auprès des élèves de l'internat d'Aksarka auprès des enfants issus Negači et des Nerkagi, fait apparaître qu'ils se considèrent comme nenets, dans la mesure où ils parlent nenets. Dans le cercle familial, leurs parents utilisent aussi le nenets, mais avec les personnes de leur communauté ils parlent le khanty.

\section{Conclusion}

41 L'histoire et l'état actuel des relations entre les Khantys et les Nenets montrent que la mémoire d'un temps où ils ne pouvaient pas vivre sur un même territoire s'est estompée. Mais traditionnellement, les ressortissants de ces peuples ont maintenu des attitudes prudentes dans les relations réciproques. On rencontre encore aujourd'hui bien des superstitions et des préjugés ; par exemple, si un Nenets rêve d'un Khanty, ce n'est pas bon signe et vice-versa - pour un Khanty, voir en rêve un Nenets n'est pas de bon augure. Beaucoup expliquent ceci par le fait que les Khantys ont des pratiques religieuses plus rigoureuses et compliquées. Même à distance, si un Nenets leur a fait quelque chose de mal, ils peuvent nuire et se venger.

Ces relations peuvent être suivies de manière particulièrement claire dans un petit groupe de personnes, où l'on trouve des ressortissants des différents peuples du Jamal : Nenets, Khantys, Komis-Zyriènes et Selkoupes. Les derniers ont tendance à regarder les premiers de haut, ce qui les irrite. Souvent, Nenets et Khantys sont en compétition, alors que les Selkoupse se retrouvent souvent du côté des Nenets. En même temps, les Komi-Zyriènes peuvent soutenir le point de vue des trois autres parties, mais en présence d'un russophone, qui occupe une position supérieure, il rejoindra toujours ce dernier. Ce sont là simplement les observations de l'auteur, qui, en tant que telles, sont par nature discutables.

La frontière de l'habitat des Nenets et des Khantys n'a pas changé, mais les éleveurs de rennes khantys pénètrent de plus en plus dans la toundra. Cette tendance peut être 
suivie facilement dans le territoire de la toundra de Panaevo, où vivent des Nenets, des Khantys ainsi que des familles mixtes. Entre groupes de Nenets et de Khantys on ne note plus la fermeture qui existait dans le passé, ce qui tient sans doute à l'environnement au village, qui est pluriethnique ainsi qu'au travail commun dans le sovkhoze de Panaevsk, où les familles khantyes et nenets s'occupent ensemble des rennes. Les individus n'ont pas de préjugés négatifs les uns envers les autres, et les cultures, les langues des voisins ne sont pas rejetés. La pénétration du lexique des voisins dans la langue du quotidien reste un processus actif, mais aujourd'hui, ce sont de plus en plus les termes russes qui s'introduisent dans le langage quotidien.

La journée des éleveurs de rennes au village d'Aksarka, 2012

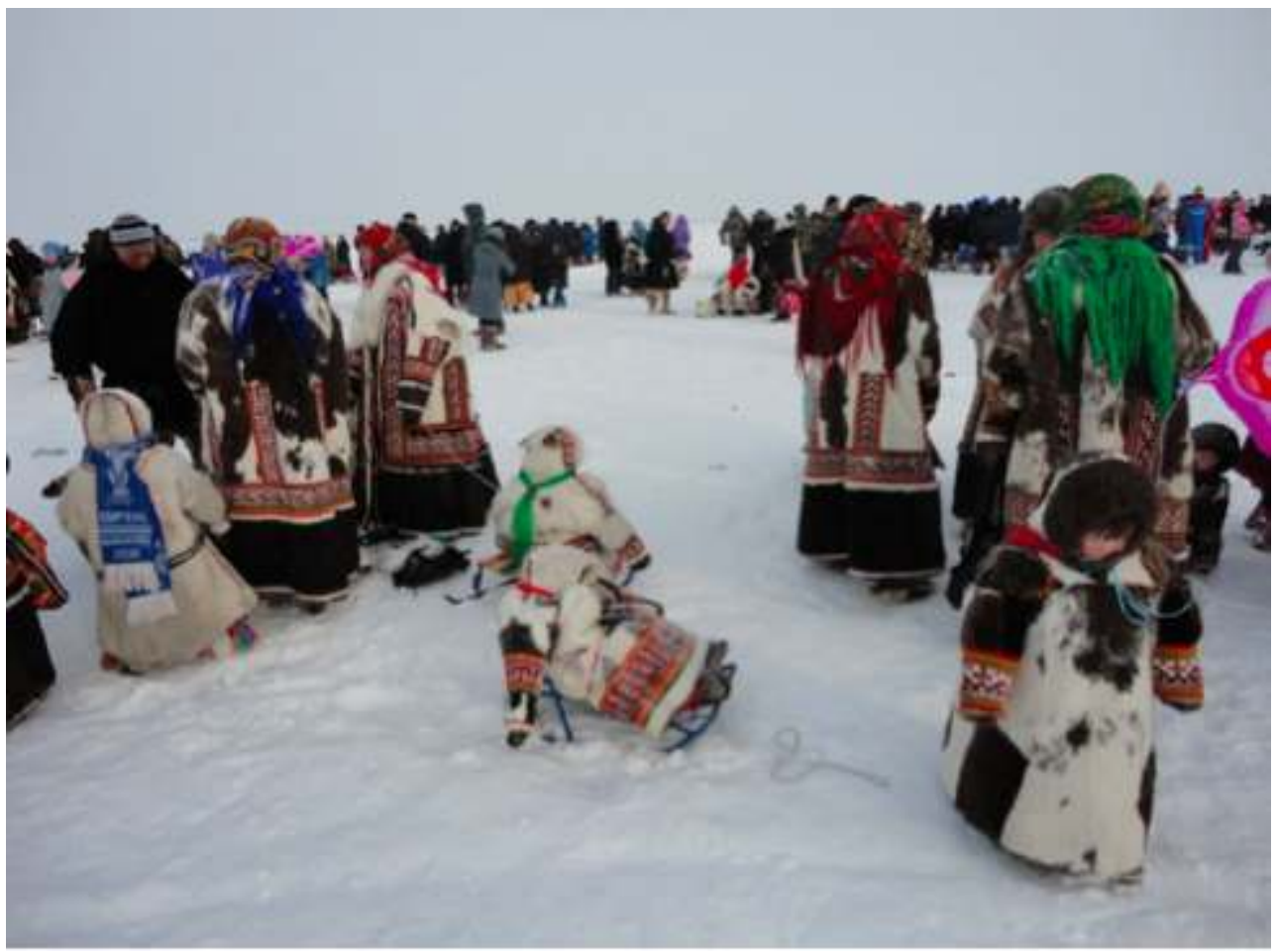

Photo Roza Laptander

\section{BIBLIOGRAPHIE}

КUŠELEVSKIJ 1868 = КУШЕЛЕВСКИЙ, Ю.И., СЕВЕРНЫЙ ПОЛЮС И ЗЕМЛЯ ЯЛМАЛ, САНКТ-ПЕТЕРБУРГ.

КUZNECOVA 2007 = КУЗНЕЦОВА, А.И., «ВАРИАТИВНОСТЬ КАК ОДИН ИЗ ФАКТОРОВ РАСШАТЫВАНИЯ ЯЗЫКОВОЙ СИСТЕМЫ В ПРОЦЕССЕ ЯЗЫКОВОГО СДВИГА (НА ПРИМЕРЕ СЕЛЬКУПСКОГО ЯЗЫКА)», ЯЗЫКОВЫЕ ИЗМЕНЕНИЯ В УСЛОВИЯХ ЯЗЫКОВОГО СДВИГА, САНКТ-ПЕТЕРБУРГ.

ОТСЕТ 1936 = ОТЧЁТ ПРИУРАЛЬСКОЙ ЭКСПЕДИЦИИ ПО ЗЕМЛЕУСТРОЙСТВУ 1933-35 ГГ., ОМСК. 
SJAZI 2000 = сЯЗИ, А.М., ОРНАМЕНТ И ВЕЩЬ В КУЛЬТУРЕ ХАНТОВ НИЖНЕГО ПРИОБЬЯ, ТОМСК: ИЗД-ВО ТОМСКОГО УН-ТА.

TEREŠČENKO 1956 = ТЕРЕЩЕНКО, Н.М., МАТЕРИАЛЫ И ИССЛЕДОВАНИЕ ПО НЕНЕЦКОМУ ЯЗЫКУ, МОСКВАЛЕНИНГРАД.

TEREŠČENKO 1959 = ТЕРЕЩЕНКО, Н.М., «К ВОПРОСУ О НЕНЕЦКО-ХАНТЫЙСКИХ ЯЗЫКОВЫХ СВЯЗЯХ», ВОПРОСЫ ЯЗЫКОЗНАНИЯ, №2.

VAHTIN 2001 = ВАХТИН, Н.Б., ЯЗЫКИ НАРОДОВ СЕВЕРА В ХХ ВЕКЕ, САНКТ-ПЕТЕРБУРГ.

ZUEV 1947 = ЗУЕВ, В.Ф., МАТЕРИАЛЫ ПО ЭТНОГРАФИИ СИБИРИ ХVIII В. (1771-1772), ЛЕНИНГРАД.

\section{NOTES}

1. Cet article a été realisé dans le cadre du projet "Socio-cultural change of Uralic languages minority in 20th-21st century Siberia analyzed through Nenets life stories" (Financé par la fondation KONE 2010, numéro 18-4665 ; subvention KONE 2011, numéro 27-6407 -- Finlande).

2. L'auteur est elle-même nenets.

3. Mot russe dépréciatif pour tous les peuples du Nord, à partir de l'ethnonyme des Tchouktches, qui faisait et font l'objet de plaisanteries les montrant comme arriérés.

4. Les arrondissements autonomes des Nenets du Iamal, des Khantys et des Mansis, Nenets, des Tchouktches (NdA). Entre 1930 et 1977, leur nom officiel était «arrondissements nationaux » (NdR).

5. Forme féminine (NdR).

6. Loh désigne 1) une personne dure d'entendement ; 2) une personne facile à tromper, une dupe $(\mathrm{NdA})$.

7. Les Nenets et les Khantys (NdA).

\section{RÉSUMÉS}

La population du nord-ouest de l'arrondissement autonome des Nenets du Jamal (Sibérie Occidentale, Russie) se compose de différents groupes ethniques. Les plus nombreux sont les Komis-Zyriènes, les Tatars sibériens et les Russes, qui sont arrivés dans cette région il y a environ trois siècles. La population autochtone se compose de Nenets, de Khantys, et de Selkoupes. Cet article, qui repose sur les locuteurs du nenets, rend compte des principales règles de communication dans les communautés multilingues. La recherche sociolinguistique sur les contacts entre les langues montre que la nature du bilinguisme et les emprunts linguistiques proviennent de la communication interethnique. Dans le cadre d'une langue ce phénomène s'actualise uniquement par des personnes bilingues qui s'identifient en même temps comme Khantys ou Nenets. Ceci est dû aux contacts permanents entre membres des deux communautés, ou lorsqu'une personne change de communauté après avoir acquis la langue voisine. La fonction sociale de ces deux ou trois langues est déterminée tout d'abord par un ensemble de facteur socioéconomiques combinés qui orientent la fréquence et la destination des passages entre une ethnicité et une autre. En même temps ces personnes, dites «migrantes ", ne perdent pas leurs 
contacts avec leur ethnie d'origine, devenant ainsi en quelque sorte médiateurs entre Khantys et Nenets et parlant les deux langues.

The population of the Northern Western part of the Yamal-Nenetskij Autonomous District (Western Siberia, Russia) is represented by diverse ethnic groups. The most numerable of them are Komi-Zyrjans, Siberian Tatars and Russians, who came to this area approximately 300 years ago. The indigenous population is represented by Nenets, Khanty, and Selkups. In this article, on the example of the Nenets language speakers, are described the common rules of the communication in the multilingual societies. The sociolinguistic research of language contacts shows that the nature of bilingualism and language borrowings arises from interethnic communication. This phenomenon is realized within one language only by bilinguals who identify themselves at the same time as Khanty or Nenets. It has happened because of regular communication between representatives of two language communities or when their members move to another community as a result of mastering the adjacent language. The social function of two or three languages stipulated, first, by the combination of socioeconomic factors that determine the frequency and direction of the transition of members of one ethnicity into another. At the same time these group of people, so-called 'migrants', do not lose contacts with their own ethnos, thus becoming some kind of 'mediators' between Khanty and Nenets groups and speak these languages.

\section{INDEX}

Mots-clés : zone frontalière, multilinguisme, bilinguisme, changement d'identité

nomsmotscles Khantys, Selkoups, Komis-Zyriènes, Russes, Nénetses, Tchouktches

Index géographique : ob' (rivière), Poluj (rivière), Jamal (raïon), Priuralskij (raïon), Bajdarata

(toundra), Salehard, Ščuč'erečje, Sob, Toupogol', Belojarsk, Ukraine

disciplines khanty, nénetse

Keywords : Border area, multilingualism, Bilingualism, identity shift

Thèmes : anthropologie, sociolinguistique 